\title{
A Case of Refractory Pulmonary Coccidioidomycosis Successfully Treated with Posaconazole Therapy
}

\author{
Patel RH' ${ }^{1}$ Pandya $S^{2}$, Nanjappa $S^{3}$ and Greene \\ J N $\mathbf{N}^{4 *}$ \\ ${ }^{1}$ Undergraduate Student, University of South Florida, \\ USA \\ ${ }^{2}$ Chief Infectious Diseases Fellow, University of South \\ Florida, Morsani College of Medicine, USA \\ ${ }^{3}$ Assistant Member, Department of Internal Hospital \\ Medicine, H. Lee Moffitt Cancer Center and Research \\ Institute, Department of Oncologic Sciences, University \\ of South Florida, Morsani College of Medicine, USA \\ ${ }^{4}$ Chief, Infectious Diseases and Hospital Epidemiologist, \\ H. Lee Moffitt Cancer Center and Research Institute, USA \\ *Corresponding author: J ohn N. Greene, Chief, \\ Infectious Diseases and Hospital Epidemiologist, H. Lee \\ Moffitt Cancer Center and Research Institute, 12902 \\ Magnolia Drive, FOB-3, Tampa, FL 33612-9497, USA
}

Received: October 14, 2017; Accepted: November 14, 2017; Published: November 21, 2017

\section{Introduction}

Coccidioidomycosis is an endemic fungal infection caused by the inhalation of arthroconidia. The two fungal species present in areas of endemicity are Coccidioides immitis and Coccidioides posadasii. These two species are present in the soil of the southwest United States, northwest Mexico, and parts of Central and South America $[1,2]$. The disturbance of endemic soil may cause airborne intrusion of highly-infectious arthroconidia from hyphae, which may cause disease through inhalation in humans and animals Generally, coccidioidomycosis can be classified as an acute, chronic and disseminated form. Patients with acute coccidioidomycosis are mostly asymptomatic and may develop complications and do not require long-term clinical monitoring. However, patients with immunosuppressive illness may present with a chronic or disseminated form which requires long-term clinical follow-up and treatment.

Pulmonary coccidioidomycosis is the most common form of the disease found in $95 \%$ of cases. Sixty percent of these cases are asymptomatic, while $40 \%$ present with an influenza-like syndrome or pneumonia [1]. Other symptoms may include fever, cough, headache, fatigue, night-sweats, and weight-loss. Rarely, patients may develop persistent pneumonia, hemoptysis, fibrocavitary infection, or cavitary complication. Infrequently, a persistent parenchymal cavity may develop and can persist along with chronic symptoms $[1,3]$. These pulmonary cavitary lesions develop in $13-15 \%$ of acute and chronic forms, however, they are uncommon in cases of disseminated disease or in immunocompromised patients $[4,5]$. They are usually present as thin- or thick-walled cavitary lesions and are generally only a single cavity $[3,6]$. They may persist for years in asymptomatic patients, but may also resolve spontaneously without the need for antifungal therapy in many cases [6].
Besides coccidioidomycosis, pneumocystis pneumonia, and Echinococcus infection have been classically associated with thinwalled cavitary lesions or nodules [3,7]. Although most cavities are asymptomatic, patients may occasionally present with symptoms of ruptured cavities which includes discomfort, bacterial super infection, or hemoptysis. Early identification and proper treatment is crucial in developing a successful treatment strategy and for avoiding complications. Diagnosis involves fungal culture, microscopy and serology testing [8]. Genomic analysis and histopathology can also be used for diagnosis of coccidioidomycosis [9]. Bronchoscopy, needle biopsy and wedge resection provides tissue specimens to aid in the confirmation of coccidioidomycosis cases.

There is an increasing prevalence of the disease in areas of endemicity, primarily in Arizona and California. Antifungal therapy is needed for chronic and disseminated coccidioidomycosis. Patients with severe pneumonia with symptoms lasting greater than four weeks may benefit from triazole antifungal therapy and require longterm clinical monitoring to ensure a successful outcome. Intravenous amphotericin B may be used in severe cases in combination with azole antifungals for 1 to 2 weeks while stabilizing the dosage of the azole antifungals [10]. Although azole antifungals are the most common option for treatment, relapse after cessation of antifungal therapy is possible [11]. Oral therapy with azole antifungals for treatment of cavitary disease may result in partial improvement [10]. Alternatively, resection via thoracotomy may be considered in patients with progressive cavity enlargement, and severe hemoptysis.

Fluconazole is the most common therapy for coccidioidomycosis. Newer triazole antifungal agents, namely posaconazole and voriconazole, have demonstrated efficacy when fluconazole is ineffective [12].
J Fam Med - Volume 4 Issue 6 - 2017

ISSN : 2380-0658 | www.austinpublishing group.com

Greene et al. (C) All rights are reserved 


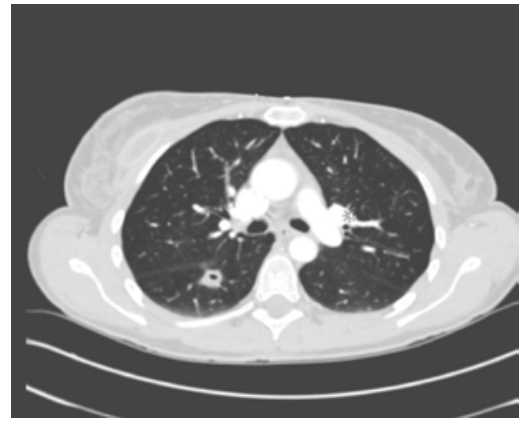

Figure 1: Computed tomography (CT) scan of the thorax with contrast depicting pulmonary cavitary lesion before surgery.

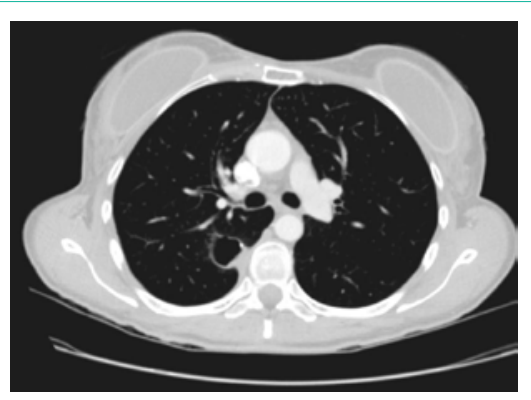

Figure 2: Computed tomography (CT) scan of the thorax with contrast depicting right posterior mid-lung thin-walled cavitary lesion with postoperative changes.

\section{Case Presentation}

A 50-year-old white female resident of Arizona presented on July of 2014 with a severe cough, hemoptysis, and influenza-like symptoms. Her symptoms started one year prior to presentation with fevers, arthralgia and a diffuse rash. She was treated for possible Lyme disease. An anticoccidioidal complement fixation titer for IgG was obtained during the initial visit which was found to be 1:32 which along with consideration of accompanying symptoms, was considered to be a sign of disseminating infection. A computed tomography of the thorax revealed a thin-walled pulmonary cavitary lesion which increased up to $5 \mathrm{~cm}$ in size until it was surgically resected (Figure 1). She underwent several diagnostic procedures including fine needle aspiration, bronchoscopy and ultimately a wedge resection of the mass which demonstrated necrotizing granuloma with positive fungal stain for Coccidioides immitis which ultimately grew on fungal cultures. She was treated with oral fluconazole $(400 \mathrm{mg} /$ day) for three months which led to slight improvement.

After fluconazole was stopped, she developed a severe cough with rash, fever, weight loss and was treated for community acquired pneumonia. She was then placed back on fluconazole $(200 \mathrm{mg})$ twice daily for approximately two months which improved her symptoms temporarily but then relapsed with symptoms of increasing fatigue, shortness of breath, dry cough and night sweats. During a followup visit in August 2015, a repeat CT scan of the thorax revealed a right posterior 3.1 by $1.7 \mathrm{~cm}$ mid-lung thin-walled cavitary lesion along with post-operative changes (Figure 2). The anticoccidioidal complement fixation titer was 1:8 and immunodiffusion was positive for IgG and IgM.

She was again placed on fluconazole (200mg/day), and noted improvement after two months. Later, she noted subjective fevers, discomfort in the right knee, migraine headaches and hair-loss which she attributed to taking fluconazole and subsequently discontinued it. Three months after stopping therapy, she again developed fatigue, joint pains, and weight-loss.

Progressive enlargement of the cavitary lesion post-operatively was also noted (Figure 3a) and posaconazole $(300 \mathrm{mg} /$ day), was prescribed. After three months, she noted significant improvement in well-being along with decreased joint-pain and fatigue. A Thoracic CT scan was performed 3-months after treatment was started and demonstrated shrinkage of the cavity to a size of $2.1 \times 1.6 \mathrm{~cm}$ (Figure 3b). A follow-up thoracic CT scan 9-month after posaconazole revealed significant shrinkage of the thin-walled cavity (Figure $3 c)$. Her overall well-being has significantly improved and she will continue posaconazole therapy indefinitely dependent on tolerability, due to the high likelihood of relapse until the cavity has completely resolved.

\section{Discussion}

With the increasing prevalence, chronicity of coccidioidomycosis, and a broad spectrum of clinical presentations, effective antifungal treatment is often necessary. The incidence of pulmonary coccidioidomycosis in endemic areas has substantially increased over the past decade. This trend has been reported beyond the traditional endemic areas of Arizona and California, due to a highly mobile society $[13,14]$. Azole antifungal therapy is the optimal choice in most patients, but relapse of infection is not uncommon.

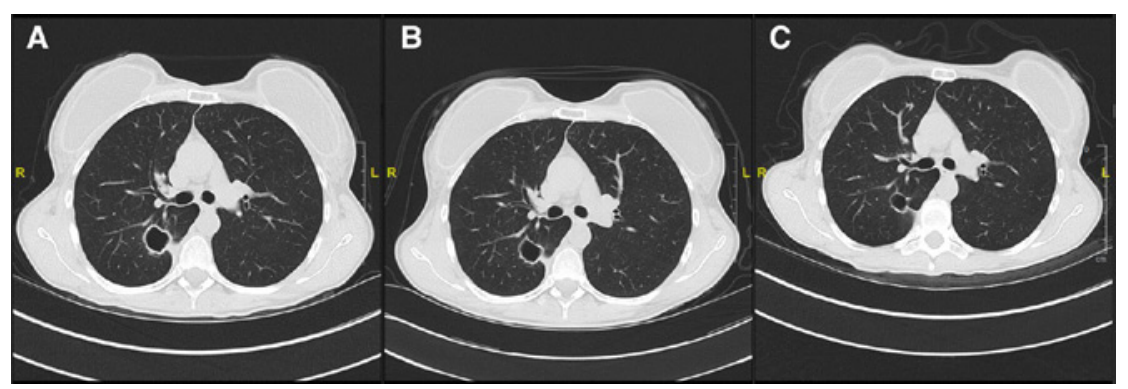

Figure 3A-C: Computed tomography (CT) scan of the thorax before and after treatment with posaconazole therapy.

A: CT of the thorax prior to treatment with posaconazole.

B: CT of the thorax after 3 months of consistent treatment with posaconazole.

C: CT of the thorax after 9 months of consistent treatment with posaconazole. 
Table 1: Common illnesses with radiographic presentation of thin-walled cavitary lesions.

Table 1: Common illnesses with radiographic presentation of thin-walled cavitary lesions.
\begin{tabular}{|c|c|}
\hline Illness & Radiographic findings \\
\hline Coccidioidomycosis & Thick or thin walled cavitary lesions with pleural effusion, single or multiple nodules, patchy opacities. \\
\hline Squamous cell carcinoma of the lung & Varying wall thickness of the cavity, pleural effusion, pulmonary nodules, hilar abnormalities. \\
\hline Blastomycosis & Smooth or thin walled cavitary lesions, patchy alveolar opacities, military pattern. \\
\hline Pulmonary histoplasmosis & Varying wall thickness of the cavity, military pattern, single or multiple nodules. \\
\hline Echinococcus & Smooth or thin walled cavitary lesions, single or multiple cystic lesions. \\
\hline Pneumocystis pneumonia & Thin walled cavities, perihilar distribution, pleural effusion, pneumatocoeles. \\
\hline Mycobacterium kansasii & Thin walled cavities, ipsilateral hilar and mediastinal lymphadenopathy, pleural effusions. \\
\hline Post-primary pulmonary tuberculosis & Varying wall thickness of the cavity, pleural effusion, lymphadenopathy, nodular opacities. \\
\hline
\end{tabular}

Posaconazole is an orally-active second-generation triazole antifungal agent that has proven to be highly effective for coccidioidomycosis based on several small studies [15]. Generally, the optimal choice for standard treatment is fluconazole or itraconazole, however, these are efficacious in $70 \%$ of cases and disease progression is common [16]. Azoles are also more effective for prophylaxis, and are generally effective in managing chronic pulmonary coccidioidomycosis. Posaconazole has shown a superior clinical efficacy to fluconazole in randomized, double-blind trials for the treatment of invasive fungal infections in immunocompromised patients. It has shown to be a stellar replacement in anti-fungal treatment for infections that are refractory to treatment with fluconazole [17]. Posaconazole was favored over itraconazole due to its decreased toxicity and increased efficacy in prophylaxis. As an alternative to azole antifungal therapy, amphotericin B may also be prescribed, however, adverse effects due to nephrotoxicity and need for intravenous administration limits its use for severe fulminant cases of coccidioidomycosis [18].

This case presented several challenges in establishing an effective mode of therapy due to multiple episodes of relapse while on fluconazole therapy. In addition, progressive enlargement of the thin-walled cavitary lung lesion, led to worsening symptoms. Unfortunately, surgery alone did not prove to be curative leading to a protracted and complicated course of illness with ongoing symptoms, and required the patient to receive prolonged antifungal therapy. The lack of specific diagnostic tests and of a standard treatment approach for all patients poses a challenge in management.

Radiographic appearance of cavitary lesions is a key characteristic in establishing a diagnosis. The most common radiographic findings for acute pulmonary coccidioidomycosis is a localized pulmonary infiltrate. Wall thickness of cavitary lesions is an important factor in determining whether the etiology of a cavity is malignant or nonmalignant. Although cavitary "thick walled" nodules are more commonly malignant than "thin walled" walled cavitary lesions, this measure cannot not be used exclusively or conclusively [7].

Thin-walled cavities have been traditionally associated with certain illnesses such as coccidioidomycosis, tuberculosis, blastomycosis, post-traumatic cysts, metastatic cavitating squamous cell carcinoma, and pulmonary histoplasmosis [19-21] (Table 1). Patients with diabetes and symptomatic coccidioidomycosis are more likely to develop cavitation. In many cases, cavities in asymptomatic patients could persist for years and resolve spontaneously without the need for antifungal therapy [22]. Complications of cavitary disease in patients suffering from coccidioidomycosis includes cavity rupture, bacterial superinfection, severe empyema, pneumothorax or a bronchopleural fistula. A smaller proportion of patients may develop chronic fibro cavitary disease which symptomatically resembles tuberculosis [7].

Compared to fluconazole, our patient displayed a significantly greater clinical response with posaconazole with less side-effects. In all, our case report presents a unique and refractory case of pulmonary coccidioidomycosis presenting as a thin walled cavitary lesion and emphasizes the importance of close clinical follow-up under triazole therapy and the efficacy of posaconazole therapy when fluconazole is inadequate or if adverse effects develop.

\section{References}

1. Spinello IM, Munoz A, Johnson RH. Pulmonary coccidioidomycosis. Semin Respir Crit Care Med. 2008; 29: 166-173.

2. Smith KM, Chawdry A, Shumyak L, et al. A Complicated Case of Coccidioidomycosis in a Patient Receiving Tumor Necrosis Factor a Inhibitor Therapy with Infliximab. Infect Dis Clin Pract. 2014; 22: 60-62.

3. Batra P. Pulmonary coccidioidomycosis. J Thorac Imaging. 1992; 7: 29-38.

4. Crum NF, Lederman ER, Stafford CM, et al. Coccidioidomycosis: a descriptive survey of a reemerging disease. Clinical characteristics and current controversies. Medicine (Baltimore). 2004; 83: 149-175.

5. Santelli AC, JE Blair, LR Roust. Coccidioidomycosis in patients with diabetes mellitus. Am. J. Med. 2006; 119: 964-969.

6. Hyde L. Coccidioidal pulmonary cavitation. Am. J. Med. 2006; 25: 890-897.

7. Gadkowski LB, Stout JE. Cavitary Pulmonary Disease. Clinical Microbiology Reviews. 2008; 21: 305-333.

8. Ampel NM. The diagnosis of coccidioidomycosis. F1000 Medicine Reports. 2010; $2: 2$.

9. Garcia SCG, Alanis JCS, Flores MG, Gonzalez SEG, Cabrera LV, Candiani JO. Coccidioidomycosis and the skin: a comprehensive review. Anais Brasileiros de Dermatologia. 2015; 90: 610-619.

10. Galgiani JN, Ampel NM, Blair JE, et al. Coccidioidomycosis. Clin Infect Dis. 2005; 41: 1217-1223.

11. Galgiani JN, Catanzaro A, Cloud GA, et al. Comparison of oral fluconazole and itraconazole for progressive, nonmeningeal coccidioidomycosis. A randomized, double-blind trial. Mycoses Study Group. Ann Intern Med. 2000; 133: 676-686.

12. Lutz JE, Clemons KV, Aristizabal BH, Stevens DA. Activity of the triazole $\mathrm{SCH} 56592$ against disseminated murine coccidioidomycosis. Antimicrob Agents Chemother. 1997; 41: 1558-1561.

13. Centers for Disease Control and Prevention (CDC). Increase in reported coccidioidomycosis: United States, 1998-2011. MMWR Morb Mortal Wkly Rep. 2013; 62: 217-221. 
14. Arizona Department of Health Services. Arizona-valley fever report (December, 2007). 2007.

15. Nagappan V, Deresinski S. Reviews of anti-infective agents: posaconazole: a broad-spectrum triazole antifungal agent. Clin Infect Dis. 2007; 45: 16101617.

16. Galgiani J. Coccidioides species. In: Mandell, Douglas, and Bennett's principles and practice of infectious diseases. Philadelphia, PA: Churchill Livingstone Elsevier. 2010; 3333-3344.

17. Greer ND. Posaconazole (Noxafil): A new triazole antifungal agent Proceedings (Baylor University Medical Center). 2007; 20: 188-196.

18. Johnson RH, Einstein HE. Amphotericin B and coccidioidomycosis. Ann N Y Acad Sci. 2007; 1111: 434-441.
19. Sheflin JR, Campbell JA, Thompson GP. Pulmonary blastomycosis: Findings on chest radiographs in 63 patients. AJR Am J Roentgenol. 1990; 154: 1177 1180.

20. Kennedy CC, Limper AH. Redefining the clinical spectrum of chronic pulmonary histoplasmosis: A retrospective case series of 46 patients. Medicine (Baltimore). 2007; 86: 252-258.

21. Greendyke WH, Resnick DL, Harvey WC. The varied roentgen manifestations of primary coccidioidomycosis. Am J Roentgenol Radium TherNucl Med. 1970; 109: 491-499.

22. Parkar AP, Kandiah P. Differential Diagnosis of Cavitary Lung Lesions. Journal of the Belgian Society of Radiology. 2016; 100: 100.
J Fam Med - Volume 4 Issue 6 - 2017

ISSN : 2380-0658 | www.austinpublishinggroup.com

Greene et al. () All rights are reserved
Citation: Patel RH, Pandya S, Nanjappa S and Greene JN. A Case of Refractory Pulmonary Coccidioidomycosis Successfully Treated with Posaconazole Therapy. J Fam Med. 2017; 4(6): 1130. 